\title{
Turbulence and Multiscaling in the Randomly Forced Navier-Stokes Equation
}

\author{
Anirban Sain, ${ }^{1}$ Manu, ${ }^{2, *}$ and Rahul Pandit ${ }^{1, \dagger}$ \\ ${ }^{1}$ Department of Physics, Indian Institute of Science, Bangalore 560 012, India \\ ${ }^{2}$ School of Physical Sciences, Jawaharlal Nehru University, New Delhi 110 067, India
}

\begin{abstract}
We present a pseudospectral study of the randomly forced Navier-Stokes equation (RFNSE) stirred by a stochastic force with zero mean and a variance $\sim k^{4-d-y}$, with $k$ the wave vector and the dimension $d=3$. We provide the first evidence for multiscaling of velocity structure functions for $y \geq 4$. We extract the multiscaling exponent ratios $\zeta_{p} / \zeta_{2}$ by using extended self-similarity, examine their dependence on $y$, and show that, if $y=4$, they are in agreement with those obtained for the Navier-Stokes equation forced at large spatial scales (3DNSE). Also well-defined vortex filaments, which appear clearly in studies of the 3DNSE, are absent in the RFNSE. [S0031-9007(98)07657-1]
\end{abstract}

Kolmogorov's classic work (K41) on homogeneous, isotropic fluid turbulence focused on the scaling behavior of velocity $\mathbf{v}$ structure functions $S_{p}(r)=\langle| \mathbf{v}_{i}(\mathbf{x}+\mathbf{r})-$ $\left.\left.\mathbf{v}_{i}(\mathbf{x})\right|^{p}\right\rangle$, where the angular brackets denote an average over the statistical steady state [1]. He suggested that, for separations $r \equiv|\mathbf{r}|$ in the inertial range, which is substantial at large Reynolds numbers Re and lies between the forcing scale $L$ and the dissipation scale $\eta_{d}$, these structure functions scale as $S_{p} \sim r^{\zeta_{p}}$, with $\zeta_{p}=p / 3$. Subsequent experiments [2] have suggested instead that multiscaling obtains with $p / 3>\zeta_{p}$, which turns out to be a nonlinear, monotonically increasing function of $p$; this has also been borne out by numerical studies of the three-dimensional Navier-Stokes equation forced at large spatial scales (3DNSE) [2,3]. The determination of the exponents $\zeta_{p}$ has been one of the central, but elusive, goals of the theory of turbulence. One of the promising starting points for such a theory is the randomly forced NavierStokes equation (RFNSE) [4-6], driven by a Gaussian random force whose spatial Fourier transform $\mathbf{f}(\mathbf{k}, t)$ has zero mean and a covariance $\left\langle\mathbf{f}_{i}(\mathbf{k}, t) \mathbf{f}_{j}\left(\mathbf{k}^{\prime}, t^{\prime}\right)\right\rangle=$ $A k^{4-d-y} P_{i j}(\mathbf{k}) \delta\left(\mathbf{k}+\mathbf{k}^{\prime}\right) \delta\left(t-t^{\prime}\right) ; \quad$ here $\mathbf{k}, \mathbf{k}^{\prime}$ are wave numbers, $t, t^{\prime}$ times, $i, j$ Cartesian components in $d$ dimensions, and $P_{i j}(\mathbf{k})$ the transverse projector which enforces the incompressibility condition. One-loop renormalization-group (RG) studies of this RFNSE yield [4,5] a K41 energy spectrum, namely, $E(k) \sim k^{2} S_{2}(k) \equiv k^{2}\left\langle|\mathbf{v}(\mathbf{k})|^{2}\right\rangle \sim k^{-5 / 3}$, if we set $d=3$ and $y=4$; this has also been verified numerically [6]. Nevertheless, these RG studies have been criticized for a variety of reasons [7,8] such as using a large value for $y$ in a small-y expansion and neglecting an infinity of marginal operators (if $y=4$ ). These criticisms of the approximations used in these studies might well be justified, but they clearly cannot be used to argue that the RFNSE is in itself inappropriate for a theory of turbulence. It is our purpose here to test whether structure functions in the RFNSE display the same multiscaling as in the 3DNSE for some value of $y$; if so, then the RFNSE can, defensibly, be used to develop a statistical theory of inertial-range multiscaling in homogeneous, isotropic fluid turbulence.

We have carried out an extensive pseudospectral study of the RFNSE and compared our results with earlier numerical studies [3,9] of the 3DNSE and experiments [2]. We find several interesting and new results: We show that structure functions in the RFNSE display multiscaling for $y \geq 4$. We obtain $\zeta_{2}$ from $S_{2}(k)$ (Fig. 1) and the exponent ratios $\zeta_{p} / \zeta_{2}$ by using the extended-self-similarity (ESS) procedure (Fig. 2a) [9,10]. We find that $\zeta_{p} / \zeta_{2}$ is close to the 3DNSE result (Fig. 2b) for $y=4$ at least for $p \leq 7$. Furthermore we show that the qualitative behaviors of the probability distributions $P\left(\delta v_{\alpha}(r)\right)$, where $\delta v_{\alpha}(r) \equiv v_{\alpha}(\mathbf{x})-v_{\alpha}(\mathbf{x}+\mathbf{r})$, are similar in the two models (Fig. 2c), but the shapes of constant- $|\omega|$

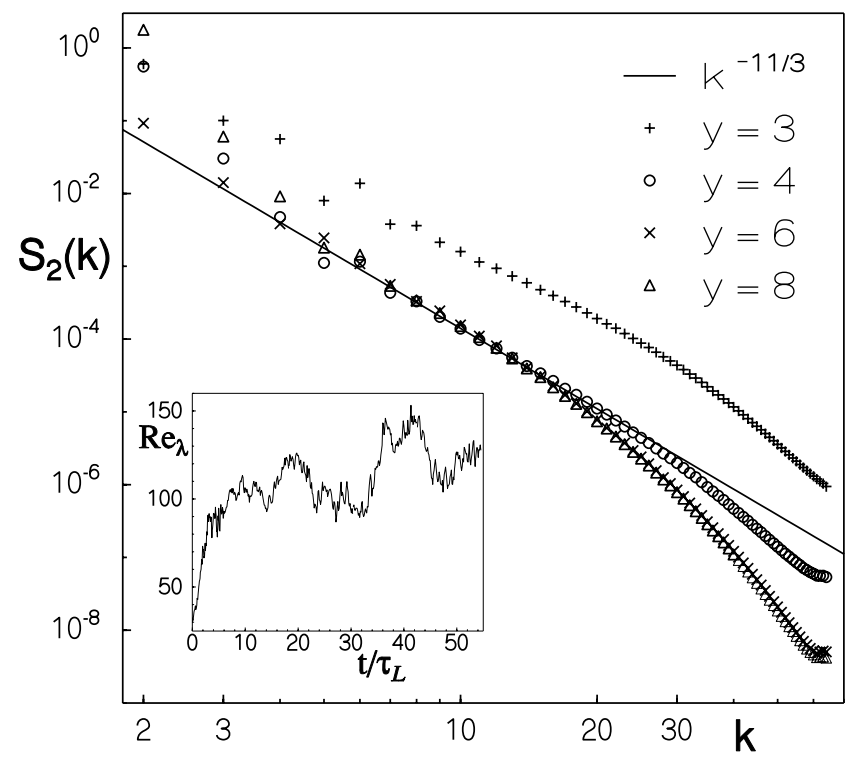

FIG. 1. Log-log plots (base 10) of $S_{2}(k)$ versus $k$ for different values of $y$. The line indicates the $\mathrm{K} 41$ result $S_{2}(k) \sim k^{-11 / 3}$. $\mathbf{k}$ indicates the shell number, which is twice the wave number $\left(=\frac{2 \pi}{L} n\right)$. The inset shows a representative plot of $\operatorname{Re}_{\lambda}$ versus time $(t)$ for $y=4$. 

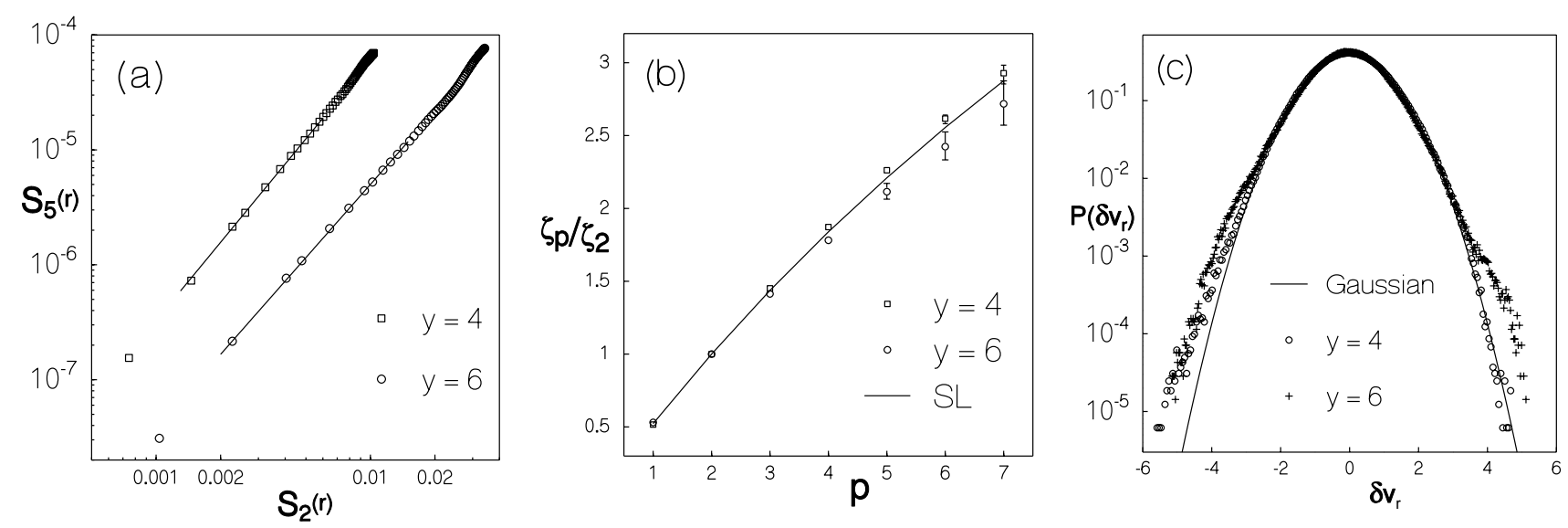

FIG. 2. (a) Log-log plots (base 10) of $S_{5}(r)$ versus $S_{2}(r)$ illustrating the ESS procedure; full lines indicate fits to points in the extended inertial range; (b) inertial-range exponent ratios $\zeta_{p} / \zeta_{2}$ versus $p$ for the RFNSE with $y=4$ and 6 [extracted from plots such as (a)]; the line indicates the SL formula; (c) semilog plots of the distribution $P\left(\delta v_{r}\right)$ [i.e., $P\left(\delta v_{\alpha}(r)\right.$ ] averaged over $\alpha$ for $r$ in the dissipation range and $y=4$ and 6; a Gaussian distribution is shown for comparison.

surfaces, where $\omega$ is the vorticity, are markedly different (Fig. 3); the stochastic force destroys well-defined filamentary structures that obtain in 3DNSE studies. This has implications for the She-Leveque (SL) [11] formula for $\zeta_{p}$ as we discuss below.

We use a pseudospectral method [12] to solve the RFNSE numerically on a $64^{3}$ grid with a cubic box of linear size $L=2 \pi$ and periodic boundary conditions; we have checked in representative cases that our results are unchanged if we use an $80^{3}$ grid or aliasing. Aside from the stochastic forcing [13], our numerical scheme is the same as in Ref. [9]. Our dissipation term, $\left(\nu+\nu_{H} k^{2}\right) k^{2} \mathbf{v}(\mathbf{k})$ in wave-vector $(k)$ space, includes both the viscosity $\nu$ and the hyperviscosity $\nu_{H}$; the exponents $\zeta_{p}$ are unaffected by $\nu_{H}$ if $\nu>0[9,14]$. For a fixed grid size we can attain higher Taylor-microscale Reynolds numbers $\operatorname{Re}_{\lambda}$ in the RFNSE, and hence a larger inertial range, than in the $3 \mathrm{DNSE}\left(\operatorname{Re}_{\lambda} \simeq 120\right.$ compared to $\operatorname{Re}_{\lambda} \simeq 22$ in our study), as noted earlier [6] for $y=4$. This advantage is reduced somewhat by the need to aver-

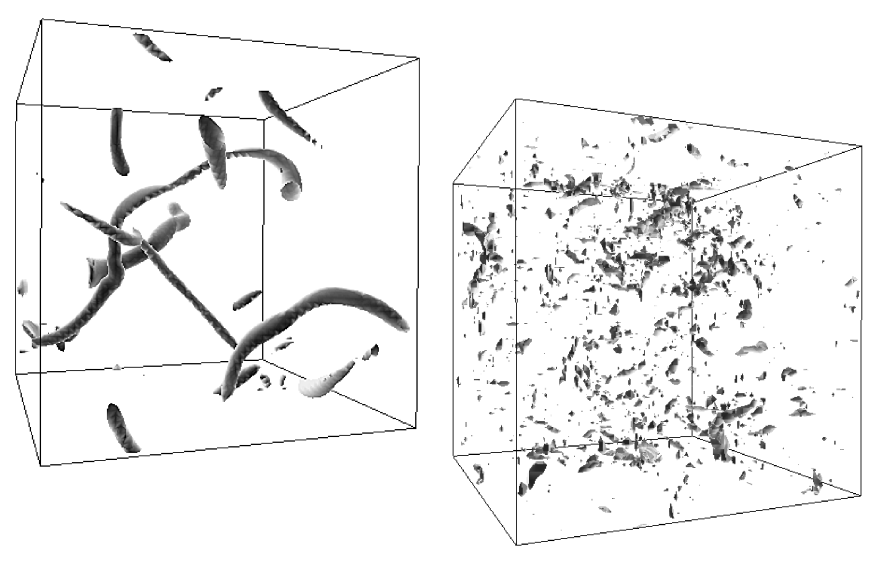

FIG. 3. Iso- $|\omega|$ surfaces obtained from instantaneous snapshots of the vorticity fields showing filaments for the 3DNSE (left) and no filaments for the RFNSE with $y=4$ (right). age statistical observables longer in the RFNSE than in the 3DNSE. In the latter case it normally suffices to average over a few box-size eddy turnover times $\tau_{L}$; this is not enough for the RFNSE since (a) $\operatorname{Re}_{\lambda}$ fluctuates strongly over time scales considerably larger than $\tau_{L}$ (inset in Fig. 1) and (b) the length of the $\mathbf{f}(\mathbf{k}, t)$ time series required to obtain a specified variance for the stochastic force is quite large $\left(\simeq 6 \tau_{L}\right.$ to achieve the given variance within $1 \%-2 \%$ ). We have collected data for averages over $(25-33) \tau_{L}$ (for different values of $y$ ), after initial transients have been allowed to decay [over times $\left.\simeq(10-20) \tau_{L}\right]$. Our $\tau_{L} \simeq 10 \tau_{I}$, the integral-scale time used in some studies [12]; $\tau_{I} \equiv L_{I} / v_{\text {rms }}$, where the integral scale $L_{I} \equiv\left[\int d k k E(k) / \int d k E(k)\right]^{-1}$ and $v_{\text {rms }}$ is the root-mean-square velocity. We have checked explicitly that the RFNSE captures the hierarchy of time scales present in the 3DNSE. In spite of the delta-correlated stochastic force in the RFNSE, the variation of $\mathbf{v}(\mathbf{k})$ as a function of time is similar in both the RFNSE and the 3DNSE: There is a hierarchy of time scales which increase with decreasing $k \equiv|\mathbf{k}|$. In the RFNSE, the stochastic force puts a high-frequency ripple on $\mathbf{v}(\mathbf{k})$ even for small $k$, but this does not affect its overall variation significantly, nor does it affect the multiscaling exponent ratios if $y=4$, as we show below.

We begin by investigating the inertial-range scaling of the $k$-space structure function $S_{2}(k) \sim k^{-\xi_{2}^{\prime}}$. Given this power-law form, the exponent $\zeta_{2}^{\prime}$ is easily related to the $r$-space exponent $\zeta_{2}$ by $\zeta_{2}=\zeta_{2}^{\prime}-3$. Our data in Fig. 1 for $4 \leq y$ are consistent with $\zeta_{2}^{\prime}=11 / 3$ [i.e., the K41 value since $\left.E(k) \sim k^{2} S_{2}(k) \sim k^{-5 / 3}\right]$. For $y=4$ this result has been reported earlier [6]. The $y$ independence of $\zeta_{2}^{\prime}$ above some critical $y_{c}$ [our data for $S_{2}(k)$ suggest $\left.y_{c} \simeq 4\right]$ is theoretically satisfying since the variance of the stochastic force in the RFNSE rises rapidly at small $k$, so we might expect that, for sufficiently large $y$, it approximates the conventional forcing of the 3DNSE at large spatial scales. This has been explored in the $N \rightarrow \infty$ 
limit of an $N$-component RFNSE [7]. This study suggests $\zeta_{2}^{\prime}=7 / 2$ for $y \geq y_{c}=4$; given our error bars (Table I) it is difficult to distinguish this from the $O(y)$ RG prediction $\zeta_{2}^{\prime}=11 / 3$ though our data are closer to the latter. For $0<y \leq 3$ both the one-loop RG [5] and the $N \rightarrow \infty$ theory [7] predict $\zeta_{2}^{\prime} \sim 1+2 y / 3+O\left(y^{2}\right)$, in fair agreement with our numerical results, especially for small $y$ (Table I). Note that, for $0<y<4$, there is no invariant energy cascade as in conventional K41: The dominance of dissipation at large $k$ does lead to an energy cascade, but the energy flux depends on the length scale $r$; specifically $\Pi(r) \approx A r^{y-4}$, with $A$ the scale-independent part of the variance of the stochastic force. A K41-type argument [15] now yields an energy-transfer rate $\sim\left\langle\delta v_{r}^{3}\right\rangle / r \sim$ $r^{(y-4)}$, whence $S_{3}(r) \sim r^{(y-3)}$ and, if we assume simple scaling as in $\mathrm{K} 41, S_{2}(r) \sim r^{(y-3) 2 / 3}$, i.e., $\zeta_{2}^{\prime}=1+2 y / 3$, as in the $O(y) \mathrm{RG}$ prediction. This formula breaks down for $y<0$; however, the RG predicts correctly that the linear-hydrodynamics result obtains in this regime.

We ensure that systematic errors do not affect $\zeta_{2}^{\prime}$ as follows. If $k_{\max }$ is the largest wave-vector magnitude in our numerical scheme, we find that $L_{I} k_{\max }$ decreases with decreasing $y$; this shortens the inertial range which can be used to obtain $\zeta_{2}^{\prime}$. The lower the value of $y$ the more difficult it is to obtain a dissipation range free of finite-resolution errors. For $y<4$, we define $k_{d} \equiv \eta_{d}^{-1}$ to be the inverse length scale at which the energy-transfer time $t_{r} \sim\left(r / v_{r}\right) \sim\left[A r^{(y-6)}\right]^{1 / 3}$ equals the diffusion time $t_{D} \sim\left[\nu k^{2}+\nu_{H} k^{4}\right]^{-1}$; this yields $\nu_{0} k_{d}^{2}+\nu_{h} k_{d}^{4}=\left[A k_{d}^{6-y}\right]^{1 / 3}$, which when solved numerically shows that, for fixed $A, k_{d}$ increases as $y$ decreases (in Table I $A$ is not fixed). Statistical steady states, with ill-resolved dissipation ranges that do not have a decaying tail [9], can be obtained by adjusting $A$. In such cases $k_{d} \gg k_{\max }$ and we get spurious results for $\zeta_{2}^{\prime}$. We find that, if we increase the hyperviscosity $\nu_{H}, k_{d}$ is sufficiently close to $k_{\max }$ so that we can resolve both inertial and dissipation ranges and obtain reliable values for $\zeta_{2}^{\prime}$. Table I shows the range over which we fit our data for $S_{2}(k)$. Since our data for $\zeta_{2}^{\prime}$ indicate that $y_{c} \simeq 4$, we investigate multiscaling only for $y \geq 4$.

Our data for $\zeta_{2}^{\prime}$ in Table I suggest that naive estimates for the $\zeta_{p}$ require longer inertial ranges than are available in our studies. However, we find that, as in the 3DNSE, the extended-self-similarity procedure $[3,9,10]$ can be used fruitfully here to extract the exponent ratios $\zeta_{p} / \zeta_{q}$ from the slopes of $\log$-log plots of $S_{p}(r)$ versus $S_{q}(r)$ (see Fig. 2) since this extends the apparent inertial range. We compare the resulting $\zeta_{p} / \zeta_{2}$ in Fig. $2 \mathrm{~b}$ with the She-Leveque formula [11], which provides a convenient parametrization for the experimental values for $\zeta_{p}$. Figure $2 \mathrm{~b}$ shows that, with $y=4$, our RFNSE exponent ratios lie very close to those for the 3DNSE and, to this extent, these two models are in the same universality class. We obtained $\zeta_{p} / \zeta_{2}$ by a regression fit. We have also checked that a local-slope analysis of ESS plots like Fig. 2a yields exponent ratios nearly indistinguishable from those shown in Fig. 2b. The error bars in Fig. 2b give a rough estimate of the systematic error associated with the choice of the precise range of points which fall in the extended inertial range; they were obtained by varying the number of points used in our regression fits. The exponent ratios for $y<4$ lie away from the 3DNSE values. One might expect naively that, at very large values of $y$, the inertial-range behaviors of structure functions of all orders should be the same as in the 3DNSE. However, strictly speaking, this is not obvious a priori, neither from renormalization-group calculations $[4,5,8]$ nor from $N \rightarrow \infty$ calculations [7]. The former are not very helpful for large $y$ since an infinity of marginal operators appears at $y=4$; all these become relevant for $y>4$. The $N \rightarrow \infty$ studies have been restricted to $p=2$. For $p>3$, our data for $\zeta_{p} / \zeta_{2}(y=6)$ fall systematically below those for $\zeta_{p} / \zeta_{2}(y=4)$ or the SL line. Also the probability distributions of $P\left(\delta v_{r}\right)$ (Fig. 2c) have nonGaussian tails for $r$ in the dissipation range, and for $y>4$ the deviations from a Gaussian distribution increase systematically with $y$. Thus, at the resolution of our calculation, the RFNSEs with $y=4$ and $y=6$ are in different universality classes. However, we point out that our data for $y=6$ are more noisy and yield a smaller inertial range $\left(k_{d} \simeq 20\right)$ than those for $y=4$ (Table I). So longer runs with finer grids might well be required to settle this issue conclusively.

Strictly speaking the RFNSE with $y=4$ falls in the same universality class as the 3DNSE only in the ESS sense. For arbitrary $y$ the energy flux through the $k$ th shell is $\Pi_{k} \equiv \Pi\left(r=k^{-1}\right) \sim \int_{1 / L}^{k}\left\langle|\mathbf{f}(\mathbf{k})|^{2}\right\rangle d^{3} k$, where $r$ is in the inertial range and we have used Novikov's theorem [15], i.e., $\langle\mathbf{f}(\mathbf{k}) \cdot \mathbf{v}(-\mathbf{k})\rangle / \sim\left\langle|\mathbf{f}(\mathbf{k})|^{2}\right\rangle$. For $\left.y\right\rangle$ 4, $\Pi_{k}$ saturates to a constant for $k L \gg 1$, but for $y=$ 4, $\Pi_{k} \sim \log (k L)$ in the RFNSE [16]. This is to be

TABLE I. The dissipation-scale wave number $k_{d}$ (see text), the integral-scale wave number $k_{I} \equiv L_{I}^{-1}$, the apparent inertial range over which we fit our data for $S_{2}(k)$, the hyperviscosities $\nu_{H}$, the exponent $\zeta_{2}^{\prime}$ that we compute, and its $O(y)$ RG value, for $1 \leq y \leq 4$. The viscosity $\nu$ is $5 \times 10^{-4}$ in all these runs which use a $64^{3}$ grid.

\begin{tabular}{ccccccc}
\hline \hline$y$ & $k_{d}$ & $k_{I}$ & Fitting range & $\nu_{H}$ & $\zeta_{2}^{\prime}$ (This study) & $\zeta_{2}^{\prime}$ from $O(y)$ RG \\
\hline 4 & 49.0 & 1.16 & $(0.1-0.5) k_{d}$ & $10^{-6}$ & $3.6 \pm 0.1$ & $\simeq 3.67$ \\
3 & 38.7 & 1.90 & $(0.16-0.52) k_{d}$ & $3 \times 10^{-6}$ & $3.0 \pm 0.1$ & $\simeq 3$ \\
2 & 35.0 & 5.90 & $(0.17-0.63) k_{d}$ & $8 \times 10^{-6}$ & $2.3 \pm 0.1$ & $\simeq 2.33$ \\
1 & 35.4 & 10.3 & $(0.2-0.7) k_{d}$ & $8 \times 10^{-6}$ & $1.6 \pm 0.15$ & $\simeq 1.67$ \\
\hline \hline
\end{tabular}


contrasted with the 3DNSE where $\Pi_{k}=$ const. Thus the inertial-range behaviors of all correlation functions in the two models are not the same. A K41-type dimensional analysis suggests that for $y=4$ the energy flux $\Pi_{k} \sim$ $\left\langle\delta v_{r}^{3}\right\rangle / r \sim \log (r / L)$; if we assume that there is no multiscaling, then $S_{p}(r) \sim[r \log (r / L)]^{p / 3}$. Multiscaling will clearly modify this simple prediction, but some weak deviation from the von Karman-Howarth form $S_{3}(r) \sim r$ must remain, since the standard derivation of this relation [15] does not go through [17] with the RFNSE result for $\Pi_{k}$. Since our data show that the ESS procedure works for the RFNSE, these weak deviations must cancel in the ratios of structure functions, and, as noted above, for $y=4$ the $\zeta_{p} / \zeta_{2}$ agree with the SL result for the 3DNSE.

Filamentary structures (Fig. 3) [18] in iso- $|\omega|$ plots are important in phenomenological models for multiscaling in fluid turbulence. For example, the SL formula [11] is obtained by postulating a hierarchical relation among the moments of the scale-dependent energy dissipation; this yields a difference equation for the exponents $\tau_{p}$, which are simply related to the exponents $\zeta_{p}$; one of the crucial boundary conditions used to solve this equation requires the codimension of the most intense structures. If these are taken to be vorticity filaments, their codimension is 2 and one gets the SL formula. Filaments have been observed in experiments also [19]. We have shown above that the exponent ratios $\zeta_{p} / \zeta_{2}$ that we obtain from the RFNSE with $y=4$ agree with the SL formula. One might expect, therefore, that filamentary structures should appear in iso- $|\omega|$ plots for the RFNSE. However, this is not the case as can be seen from the representative plot shown in Fig. 3. The stochastic forcing seems to destroy the well-defined filaments observed in the 3DNSE without changing the multiscaling exponent ratios. Therefore, the existence of vorticity filaments is not crucial for obtaining these exponents, which is perhaps why simple shell models $[9,20]$ also yield good estimates for $\zeta_{p}$.

In summary, then, we have shown that the RFNSE with $y=4$ exhibits the same multiscaling behavior as the 3DNSE, at least in the ESS sense. Probability distributions like $P\left(\delta v_{r}\right)$ (Fig. 2c) are also qualitatively similar in the two models, in so far as they show deviations from Gaussian distributions for $r$ in the dissipation range. It would be interesting to see if the RFNSE model can be obtained as an effective, inertial-range equation for fluid turbulence. We have tried to do this by a coarse-graining procedure that has been used [21] to map the Kuramoto-Sivashinsky (KS) equation onto the Kardar-Parisi-Zhang (KPZ) equation; however, it turns out that the 3DNSE $\rightarrow$ RFNSE mapping, if it exists, is far more subtle than the $\mathrm{KS} \rightarrow \mathrm{KPZ}$ mapping as we discuss elsewhere [17].

We thank C. Das, A. Pande, S. Ramaswamy, and H. R. Krishnamurthy for discussions, CSIR (India) for support, and SERC (IISc, Bangalore) for computational resources.

*Present address: Indian Institute of Foreign Trade, New Delhi, India.

${ }^{\dagger}$ Also at Jawaharlal Nehru Centre for Advanced Scientific Research, Bangalore, India.

[1] A. N. Kolmogorov, C.R. Acad. Sci. USSR 30, 301 (1941).

[2] For recent reviews, see K. R. Sreenivasan and R. Antonia, Annu. Rev. Fluid Mech. 29, 435 (1997); S. K. Dhar et al., Special issue on Nonlinearity and Chaos in Physical Sciences [Pramana J. Phys. 48, 325 (1997)].

[3] N. Cao, S. Chen, and K. R. Sreenivasan, Phys. Rev. Lett. 77, 3799 (1996).

[4] C. DeDominicis and P.C. Martin, Phys. Rev. A 19, 419 (1979); D. Forster, D. R. Nelson, and M. J. Stephen, Phys. Rev. A 16, 732 (1977).

[5] V. Yakhot and S. A. Orszag, Phys. Rev. Lett. 57, 1722 (1986); J. K. Bhattacharjee, J. Phys. A 21, L551 (1988).

[6] V. Yakhot, S. A. Orszag, and R. Panda, J. Sci. Comput. 3, 139 (1988).

[7] C. Y. Mou and P. B. Weichman, Phys. Rev. Lett. 70, 1101 (1993).

[8] G. L. Eyink, Phys. Fluids 6, 3063 (1994).

[9] S. K. Dhar, A. Sain, and R. Pandit, Phys. Rev. Lett. 78, 2964 (1997).

[10] R. Benzi et al., Phys. Rev. E 48, R29 (1993).

[11] Z. S. She and E. Leveque, Phys. Rev. Lett. 72, 336 (1994).

[12] M. Meneguzzi and A. Vincent, in Advances in Turbulence 3, edited by A. V. Johansson and P. H. Alfredsson (Springer, Berlin, 1991), pp. 211-220.

[13] For analogous studies of the randomly forced Burger's equation, see A. Chekhlov and V. Yakhot, Phys. Rev. E 51, 2739 (1995); F. Hayot and C. Jayaprakash, Phys. Rev. E 54, 4681 (1996).

[14] N. Cao, S. Chen, and Z. S. She, Phys. Rev. Lett. 76, 3711 (1996).

[15] U. Frish, Turbulence: The Legacy of A.N. Kolmogorov (Cambridge University Press, Cambridge, England, 1995).

[16] Thus at the level of $\Pi_{k}$ it seems that $y_{c}=4$, but, as noted above, this value of $y_{c}=4$ does not emerge from our data for $\zeta_{p} / \zeta_{2}(p \geq 3)$ and $P\left(\delta v_{r}\right)$.

[17] A. Sain and R. Pandit (unpublished).

[18] E. D. Siggia, J. Fluid Mech. 107, 375 (1981); Z.S. She, E. Jackson, and S. A. Orszag, Nature (London) 344, 226 (1990).

[19] S. Douady, Y. Couder, and M. E. Brachet, Phys. Rev. Lett. 67, 983 (1991).

[20] D. Pisarenko et al., Phys. Fluids A 5, 2533 (1993).

[21] C. Jayaprakash, F. Hayot, and R. Pandit, Phys. Rev. Lett. 71, 12 (1993). 